\title{
El tamoxifeno reduce la recurrencia y la mortalidad del cáncer mamario temprano con receptores estrogénicos positivos
}

Tamoxifen reduces recurrence and mortality in positive estrogen receptor early breast cancer

Early Breast Cancer Trialist Collaborative Group. Lancet 2011;378:771-84.

\section{Objetivos}

Determinar la efectividad del tamoxifeno adyuvante en pacientes con cáncer de mama temprano.

Fuente de datos y selección de estudios

Meta-análisis con datos de 21.457 pacientes individuales* (10.645 mujeres tenían receptores estrogénicos positivos (RE+) y provenían de 20 ensayos controlados aleatorizados, publicados entre 2005 a 2011, que comparan el uso de tamoxifeno vs. no tratamiento).

\section{Resultados principales}

El tamoxifeno redujo la recurrencia en pacientes con cáncer de mama RE+, pero no en aquellas con RE negativos. Entre las pacientes con $\mathrm{RE}+$, el tamoxifeno redujo $22 \%$ (IC 95\% 17 a 27 ) la mortalidad general y la mortalidad por cáncer mamario (Tabla 1). No hubo diferencias significativas en cuanto a la mortalidad por tromboembolismo de pulmón, otros cánceres, accidente cerebrovascular o muertes de causa cardiovascular. En cáncer mamario RE+, el tamoxifeno aumento (sobre todo en mujeres añosas) el riesgo de cáncer uterino (excluyendo el de cérvix) un $140 \%(P<0,001)$, aunque con un número absoluto de muertes poco relevante.

Tabla 1: Tamoxifeno adyuvante durante cinco años vs control en cáncer mamario temprano con receptores estrogénicos positivos

\begin{tabular}{|c|c|c|c|c|c|}
\hline Resultados & Seguimiento & Tamoxifeno & Control & RRR (IC95\%) & NNT (IC95\%)/P \\
\hline Recurrencia $^{\dagger}$ & $\begin{array}{c}\text { A } 15 \text { años } \\
0 \text { a } 4 \text { años } \\
5 \text { a } 9 \text { años } \\
10 \text { a } 14 \text { años }\end{array}$ & $\begin{array}{c}33 \% \\
3,7 \% \text { por año } \\
2,6 \% \text { por año } \\
2,1 \% \text { por año }\end{array}$ & $\begin{array}{c}46 \% \\
6,7 \% \text { por año } \\
3,5 \% \text { por año } \\
2,1 \% \text { por año }\end{array}$ & $\begin{array}{l}39 \% \text { (35 a 43) } \\
47 \% \text { (43 a 52) } \\
32 \%(22 \text { a } 40) \\
3 \%(-18 \text { a } 21)\end{array}$ & $\begin{array}{c}6(6 \text { a } 7) \\
<0,001 \\
<0,001 \\
\text { NS }\end{array}$ \\
\hline $\begin{array}{l}\text { Mortalidad por cáncer } \\
\text { mamario }\end{array}$ & $\begin{array}{l}\text { A } 15 \text { años } \\
0 \text { a } 4 \text { años } \\
5 \text { a } 9 \text { años } \\
10 \text { a } 14 \text { años }\end{array}$ & $\begin{array}{c}24 \% \\
1,8 \% \text { por año } \\
2,3 \% \text { por año } \\
1,5 \% \text { por año }\end{array}$ & $\begin{array}{c}33 \% \\
2,6 \% \text { por año } \\
3,2 \% \text { por año } \\
2,3 \% \text { por año }\end{array}$ & $\begin{array}{l}30 \%(25 \text { a } 36) \\
29 \%(20 \text { a } 38) \\
34 \%(25 \text { a } 42) \\
32 \%(17 \text { a } 44)\end{array}$ & $\begin{array}{c}11(9 \text { a 13) } \\
<0,001 \\
<0,001 \\
<0,001\end{array}$ \\
\hline
\end{tabular}

NS: No significativo, RRR: Reducción de Riesgo Relativo; NNT: Número necesario para tratar; IC intervalo de confianza. † 1655 pacientes (100 \% receptores hormonales positivos, $44 \%$ ganglios positivos, $51 \%$ quimioterapia)

\section{Conclusiones}

El tratamiento adyuvante con tamoxifeno durante cinco años prolonga el intervalo libre de enfermedad y la sobrevida global en pacientes pre y post-menopaúsicas RE+. Se observó un aumento del riesgo de cáncer de endometrio y una disminución en el riesgo de cáncer de mama contralateral en las mujeres tratadas.

Fuentes de financiamiento: Unidad de estudios epidemiológicos de la Universidad de Oxford.

\section{Comentario}

El cáncer de mama es el más común de todos los cánceres en la mujer y la segunda causa de muerte por cáncer. ${ }^{1}$ El $75 \%$ expresan receptores hormonales y es frecuente el diagnostico en estadios tempranos (localizado en la mama o con ganglios axilares positivos). La terapia hormonal es uno de los pilares del tratamiento tanto en la enfermedad temprana como avanzada de pacientes con receptores hormonales positivos. Como primera línea son de elección el tamoxifeno en pacientes premenopaúsicas y los inhibidores de aromatasa (anastrazol, letrozol) en pacientes post-menopaúsicas. La combinación de estos tratamientos y nuevas terapias han demostrado beneficio en pacientes progresadas (exemestane, fulvestrant) ${ }^{2-3}$. El desafío es lograr articular un plan de tratamiento que incluya todas las alternativas disponibles (cirugía, quimioterapia, hormonoterapia, terapias blanco) que se amolden a la situación de la paciente.

\section{Conclusiones del comentador}

Por eficacia, costo-efectividad y seguridad, el tamoxifeno sigue siendo la base del tratamiento para las mujeres con cáncer de mama RE+ que son pre o peri-menopáusicas o con amenorrea inducida por la quimioterapia. Ésta es la mejor evidencia al momento sobre la primera terapia de blanco descripta en el cáncer de mama: la hormonoterapia.

Federico Losco [ Especialista en Clínica Médica. Residente en Oncología Clínica del Instituto Alexander Fleming fedelosco@gmail.com ]

Losco F. El tamoxifeno reduce la recurrencia y la mortalidad del cáncer mamario temprano con receptores estrogénicos positivos. Evid Act Pract Amb. Ene-Mar 2013. 16(1).5. Comentado de: Early Breast Cancer Trialists' Collaborative Group. Relevance of breast cancer hormone receptors and other factors to the efficacy of adjuvant tamoxifen: patient-level meta-analysis of randomised trials. Lancet. 2011;378:771-84. PMID: 21802721.

\section{Referencias}

1. Jemal A, Bray F. Center MM, Et al. CA Cancer J Clin 2011:61:69-90

2. Michael Gnant, Nadia Harbeck, Christoph Thomssen. Breast Care (Basel) 2011. 6(2): 136-141

3. NCCN Clinical Practice Guidelines in Oncology (2013). De http://www.nccn.org/professionals/physician_gls/f_guidelines.asp. 


\section{Efectos de la reducción del colesterol LDL con estatinas en pacientes con bajo riesgo cardiovascular}

The effects of lowering of lowering LDL cholesterol with statin therapy in people at low risk of vascular disease

\section{Objetivo}

Conocer los efectos de reducir el colesterol LDL (c-LDL) con estatinas en pacientes con bajo riesgo de enfermedad cardiovascular (CV) a cinco años.

Diseño, fuentes de datos, selección de estudios y extracción de Datos

Meta-análisis a través del protocolo de búsqueda y elegibilidad de los estudios de la Colaboración "Cholesterol Treatment Trialist". Incluyó el análisis de datos individuales de 27 ensayos clínicos con un total de 174.149 pacientes. 22 ensayos habían comparado la eficacia del tratamiento con estatinas con controles $(n=134.537)$, con una mediana de seguimiento de 4,8 años, una media de c-LDL basal de $143 \mathrm{mg} / \mathrm{dL}$ y una diferencia media de $42 \mathrm{mg} / \mathrm{dL}$ al año. Los cinco ensayos restantes habían comparado diferentes dosis (altas versus bajas) de tratamientos con estatinas $(n=39.612)$, con una mediana de seguimiento de 5,1 años, una media basal de 98 $\mathrm{mg} / \mathrm{dL}$, y una diferencia media de $20 \mathrm{mg} / \mathrm{dL}$ al año.

\section{Medidas de resultado y análisis}

Los resultados primarios fueron los principales eventos CV: eventos coronarios mayores (infarto agudo de miocardio no fatal o muerte coronaria), accidente cerebrovascular (ACV) isquémico o hemorrágico, procedimientos de revascularización coronaria, cáncer y mortalidad por causas específicas.

El análisis se realizó por intención de tratar, asignandose a los participantes de cada estudio a una de cinco categorías de riesgo CV mayor a cinco años (menor a 5\%; entre 5 y $10 \%$; entre 10 y $20 \%$; entre 20 y 30\%; mayor a 30\%), y realizándose el análsis estadísitico a través del modelo de riesgo proporcional de Cox.
Cholesterol Treatment Trialist Collaborators. Lancet 2012;380:581-90.

\section{Resultados principales}

La incidencia media de eventos coronarios fue de $2,6 \%$ y la de otros eventos CV mayores, de 3\%.

En los 27 ensayos el uso de estatinas se asoció a una incidencia de eventos CV de $21 \%$ por cada $39 \mathrm{mg} / \mathrm{dL}$ de disminución del c-LDL (RR 0,79; IC95\% 0,77 a 0,81), observándose reducciones significativas en todas las categorías de riesgo analizado, independientemente de la edad, el sexo, el c-LDL basal y el antecendente de enfermedad CV previa, diabetes o enfermedad renal crónica.

No hubo evidencia de un aumento de cáncer o de muerte por cáncer o por otra causa no CV.

El tratamiento con estatinas se asocia a algunos riesgos como el aumento de la incidencia de miopatía (riesgo atribuible de 50 cada 100.000 personas tratadas durante cinco años) y raramente de rabdomiólisis (10 cada 100.000), así como un probable aumento del riesgo de ACV hemorrágico (50 por cada 100.000). Sin embargo, sus beneficios superarían los riesgos, especialmente teniendo en cuenta la incidencia de miopatía es dosis-dependiente y que el aumento del riesgo de ACV hemorrágico se vería compensado por la reducción del riesgo de los isquémicos.

\section{Conclusiones}

La disminución del c-LDL con estatinas redujo significativamente el riesgo de eventos $\mathrm{CV}$ mayores en pacientes con bajo riesgo $\mathrm{CV}$ (menor a 10\% a cinco años). Por cada mil pacientes que lograron un descenso de $39 \mathrm{mg} / \mathrm{dL}$ de sus niveles de c-LDL durante cinco años, 11 de ellos evitaron un evento CV (NNT=91).

Fuente de financiamiento: British Heart Foundation; UK Medical Research Council; Cancer Research UK; European Community Biomed Programme; Australian National Health and Medical Research Council; National Heart Foundation, Australia. La mayoría de los ensayos evaluados fueron financiados por la industria farmacéutica.

\section{Comentario}

Este estudio documentó la eficacia y la seguridad del tratamiento con estatinas para la reducción de la incidencia de eventos CV mayores en pacientes con un riesgo CV menor a $10 \%$ a cinco años, a través de una reducción del c-LDL de 39 $\mathrm{mg} / \mathrm{dL}$, habitualmente alcanzable con los regímenes disponibles. Dado que es necesario que 91 personas de bajo riesgo reciban estatinas durante cinco años para que una de ellas evite un evento $\mathrm{CV}$, un punto pendiente a discutir es ¿qué piensan nuestros pacientes?.

Siguiendo esta línea de pensamiento, sería razonable discutir con ellos sus preferencias, fomentando las decisiones compartidas.

\section{Coclusiones de la comentadora}

Quedan pendientes algunas preguntas vinculadas a nuestra práctica clínica a las decisiones a ser tomadas desde la perspectiva de la salud pública: ¿luego de estos resultados se sustenta el uso rutinario de estatinas en prevención primaria de rutina?, ¿cuál sería la adherencia a su uso y la tolerancia en personas con bajo riesgo?, ¿en qué momento comenzaríamos a indicarlas y hasta cuándo? ¿cuál sería su costo-efectividad y/o su impacto presupuestario en Sudamérica?

María Florencia Grande Ratti [ Servicio de Medicina Familiar y Comunitaria del Hospital Italiano de Buenos Aires. maria.grande@ hospitalitaliano.org.ar ]

Grande Ratti MF. Efectos de la reducción del colesterol LDL con estatinas en pacientes con bajo riesgo cardiovascular. Evid Act Pract Amb. Ene-Mar 2013. 16(1).6. Comentado de: Cholesterol Treatment Trialist Collaborators. The effects of lowering of lowering LDL cholesterol with statin therapy in people at low risk of vascular disease: meta-analysis of individual data from 27 randomised trials. Lancet 2012;380:581-90. PMID:22607822.

Referencias

1. Cholesterol Treatment Trialists' (CTT) Collaboration. Protocol for a prospective collaborative overview of all current and planned randomized trials of cholesterol treatment regimens. Am J Cardiol. 1995 Jun 1;75(16):1130-4 